\title{
On the statistical theory of visco-elastic properties of electrolyte solutions
}

\author{
S.Odinaev, A.Dodarbekov \\ Physical-Technical Institute of the Academy of Sciences of Tadjikistan, \\ 229/1 Ajni Str., 734063 Dushanbe, Tadjikistan
}

Received August 5, 2000

Based on the generalized kinetic equation for single and pair correlation distribution functions, analytical expressions for dynamic coefficients of shear and bulk viscosity and corresponding elastic modulus are obtained. The frequency dispersion of these coefficients and elastic modulus are caused mainly by translational and structural relaxation processes. The asymptotic behaviour of these expressions at low and high frequency is investigated.

Key words: kinetic equation, translational and structural relaxation, shear and bulk viscosity, elastic modulus, electrolyte solutions

PACS: $61.20 . \mathrm{Qg}, 66.10 . \mathrm{Ed}$

Since electrolyte solutions are widely used, we need to know their viscoelastic, thermoelastic, acoustic and electroconductive properties. A number of theoretical and experimental works are devoted to the study of viscoelastic properties of solutions. An especially relevant task is taking into account the modification of viscosity stimulated by the following factors: contributions of the solution structure, hydration, charge and size of ions causing a modification of the solution structure as well as the process of restoring the solution equilibrium (relaxation).

In works $[1,2]$, starting from the kinetic equations for single and pair distribution functions, expressions for static transport coefficients of a binary solution are obtained which are expressed by means of molecular parameters and a pair distribution function. In [3] the frequency dependence of the transport coefficients is generalized by Zwanzig in the case of binary mixtures. The main attention is paid to the research of the contribution of internal degrees of freedom to bulk viscosity and dependence of transport coefficients on concentration. The viscosity coefficients, obtained in [4] are presented as integrals of time correlation functions and their determination is a difficult task.

It is known, that similar to the static case for the frequency dependence, the bulk viscosity is many times larger than shear viscosity, and its magnitude is determined by interactions of structural units of a solution. In water and in an aqueous solution 
the bulk viscosity arises mainly because of structural relaxation processes [5]. The theoretical calculations made by Fisher with co-workers [6] show that the hydration of ions changes the bulk viscosity four times more than the shear one. The authors explain by the fact that an essential moment for hydration is the compression of water under the influence of the electrical field of ions.

The main achievement of the statistical theory of the transport and elastic properties of electrolyte solutions is the definition of kinetic factors and modules of elasticity in two limiting cases of slow and fast processes. In the main, the existing theories start from the offer concerning a single characteristic relaxation time of kinetic factors which is not enough for the description of the processes of structural relaxation. Moreover, the knowledge of frequency-dependent factors of transport and modules of elasticity with considering the contribution of a structural relaxation, will permit to investigate theoretically such acoustic properties of solutions as a variance of a sound velocity, a sound absorption coefficient, as well as spectrum of collective modes. The present work is devoted to a research of viscoelastic properties of electrolyte solutions based on the uniform microscopic theory with considering the contribution of various relaxational processes.

We consider a single-phase electroneutral inhomogeneous system. $N_{a}, m_{a}, d_{a}$ and $N_{b}, m_{b}, d_{b}$ are the numbers, masses and diameters of structural units of a solution of the sorts $a$ and $b$, respectively. The particles of solution interact by means of spherically-symmetrical potential $\Phi_{a b}(|\vec{r}|), \vec{r}_{a b}=\vec{q}_{2}-\vec{q}_{1}$ is a distance between the particles. Let us assume that the solvent is a neutral medium, offering resistance to the driven ions of the soluted substance and results in hydration.

In the case of small deviation of the solution from an equilibrium (in linear approximation) the stress tensor is determined microscopically as follows [7]:

$$
\sigma^{\alpha \beta}\left(q_{1}, t\right)=-\sum_{a}\left(\stackrel{k}{P} \delta^{\alpha \beta}+K_{a}^{\alpha \beta}\right)+\frac{1}{2} \sum_{a} \sum_{b} \int \frac{\partial \Phi_{a b}}{\partial r} \cdot \frac{r^{\alpha} r^{\beta}}{r} n_{a b} \mathrm{~d} \vec{r}
$$

where

$$
\vec{r}=\vec{r}_{a b} / d_{a b}
$$

is the reduced distance between particles of the solution,

$$
d_{a b}=\frac{1}{2}\left(d_{a}+d_{b}\right)
$$

and

$$
\stackrel{k}{P}_{a}\left(\vec{q}_{1}, t\right)=\frac{1}{3} \int \frac{\tilde{P}_{a}^{2}}{m_{a}} f_{a} \mathrm{~d} \vec{p}_{a}
$$

is the kinetic part of pressure,

$$
K_{a}^{\alpha \beta}\left(\vec{q}_{1}, t\right)=\frac{1}{m_{a}} \int\left(\tilde{P}_{a}^{\alpha} \tilde{P}_{a}^{\beta}-\frac{1}{3} \tilde{P}_{a}^{2} \delta^{\alpha \beta}\right) f_{a} \mathrm{~d} \vec{p}_{a}
$$

is the kinetic part of the viscous stress tensor,

$$
n_{a b}\left(\vec{q}_{1}, \vec{q}_{2}, t\right)=\int f_{a b} \mathrm{~d} \vec{p}_{a} \mathrm{~d} \vec{p}_{b}
$$


is the binary density,

$$
\tilde{P}_{a}^{\alpha}=p_{a}^{\alpha}-m_{a} v^{a}\left(\vec{q}_{1}, t\right)
$$

is the relative impulse of a particle of the sort $a$.

Using the kinetic equations for $f_{a}$ and $f_{a b}$, which consider the contribution of spatial density correlation and velocity correlation [7] in the framework of superposition approximation for the three-particle distribution function $f_{a b c}$, for the $P_{a}^{k}$ and $K_{a}^{\alpha \beta}$ one obtains:

$$
\begin{gathered}
\frac{\partial \stackrel{k}{P} a}{\partial t}+\frac{5}{3} \stackrel{k}{P}_{a}(0) \operatorname{div} \vec{v}+\frac{2}{3} \frac{\partial \stackrel{k}{S}_{a}^{\alpha}}{\partial g_{1}^{2}}-\frac{2}{3} \sum_{b} \int \frac{\partial \phi_{a b}}{\partial r^{\alpha}} J_{2(a)}^{\alpha} \mathrm{d} \vec{r}=0 \\
\frac{\partial K_{a}^{\alpha \beta}}{\partial t}+22_{a}^{k}(0)\left\{\frac{\partial v^{a}}{\partial q_{1}^{\beta}}\right\}+2\left\{\frac{\partial \stackrel{k}{S}_{a}^{\alpha}}{\partial q_{1}^{\beta}}\right\}-2 \sum_{b} \int\left\{\frac{\partial \phi_{a b}}{\partial r^{\alpha}} J_{2}^{\beta}(a)\right\} \mathrm{d} \vec{r}=-\frac{2 \beta_{a}}{m_{a}} K_{a}^{\alpha \beta} .
\end{gathered}
$$

For a perturbed part of a binary density we shall receive the Smoluchowski equation. Its solution has a form:

$$
n_{a b}^{\prime}=\int_{0}^{t} \mathrm{~d} t_{1} \int_{-\infty}^{\infty} G_{a b}\left(r, r_{1}, t-t_{1}\right) F_{a b}\left(\vec{g}_{1}, \vec{r}_{1}, t_{1}\right) \mathrm{d} \vec{r}_{1},
$$

where $G_{a b}\left(r, r_{1}, t-t_{1}\right)$ is the solution to the homogeneous Smoluchowski equation which can be written in the radial-symmetrical case as:

$$
\begin{aligned}
& G_{a b}=\frac{2\left(r r_{1}\right)}{(2 \pi)^{3}}\left[\pi / \omega_{a b}\left(t-t_{1}\right)\right]^{-1 / 2}\left\{\exp \left[-\frac{\left(r-r_{1}\right)^{2}}{4 \omega_{a b}\left(t-t_{1}\right)}\right]-\exp \left[-\frac{\left(r+r_{1}\right)^{2}}{4 \omega_{a b}\left(t-t_{1}\right)}\right]\right\}, \\
& F_{a b}=-\varphi_{a b} \operatorname{div} \vec{v}-n_{a} n_{b}\left(r^{a} r^{b}-\frac{1}{3} r^{2} \delta^{\alpha \beta}\right) \frac{\partial \ln g_{a b}}{\partial \ln r}\left\{\frac{\partial v^{a}}{\partial q_{1}^{\beta}}\right\} \\
& -\left(n_{a} n_{b} / d_{a b}\right)\left(\frac{e_{b}}{\beta_{b}}-\frac{e_{a}}{\beta_{a}}\right) \frac{\partial \ln g_{a b}^{0}}{\partial r} \frac{r^{a}}{r} E^{a}, \\
& \varphi_{a b}=2 n_{a} n_{b} g_{a b}^{0}\left\{1+\frac{1}{6} \frac{\partial \ln g_{a b}^{0}}{\partial \ln r}-\frac{1}{2}+\left[\left(\frac{\partial \ln g_{a b}^{0}}{\partial \ln n}\right)_{t}+\gamma\left(\frac{\partial \ln g_{a b}^{0}}{\partial \ln T}\right)_{n}\right]\right\}, \\
& \gamma=\frac{1}{n C_{\nu}}\left(\frac{\partial P}{\partial T}\right)_{n} \\
& \left\{\frac{\partial M^{\alpha}}{\partial q \beta}\right\}=\frac{1}{2}\left(\frac{\partial M^{\alpha}}{\partial q \beta}+\frac{\partial M^{\beta}}{\partial q \alpha}-\frac{2}{3} \delta^{\alpha \beta} \operatorname{div} \vec{M}\right), \quad M^{\alpha}=v^{\alpha}, \stackrel{k}{S}_{a}^{\alpha} \text { etc. } \\
& \omega_{a b}=\frac{k T}{d_{a b}^{2}}\left(\frac{\beta_{a}+\beta_{b}}{\beta_{a} \beta_{b}}\right),
\end{aligned}
$$

$\beta_{a}, \beta_{b}$ are the friction coefficients, $g_{a b}^{0}(r)$ is the equilibrium radial distribution function, $n_{a}$ and $n_{b}$ are the number density of particles of sorts $a$ and $b$, respectively, $\stackrel{k}{S}_{a}^{\alpha}$ is the kinetic part of heat of sort $a$. 
Substituting (4) and also solutions (2) and (3) in case of independent flows into (1), performing the Fourier-transformation on time, we get for the dynamic modules of elasticity and viscosity coefficients:

$$
\begin{aligned}
K(\omega)= & K_{s} \\
& +\left(\rho \frac{N_{0}}{\mu}\right)^{2} \sum_{a} \sum_{b} \frac{2 \pi}{3} C_{a} C_{b} d_{a b}^{3} \omega \int_{0}^{\infty} \mathrm{d} r r^{2} \frac{\partial \Phi_{a b}}{\partial r} \int_{0}^{r} G_{2}^{a b}\left(r, r_{1}, \omega\right) \stackrel{\varphi}{\varphi}_{a b} r_{1} \mathrm{~d} r_{1}, \\
\mu(\omega)= & \frac{\sum_{a} P_{a}^{k}(0)\left(w \tau_{a}\right)^{2}}{1+\left(\omega \tau_{\phi}\right)^{2}} \\
& +\left(\rho \frac{N_{0}}{M}\right)^{2} \sum_{a} \sum_{b} \frac{2 \pi}{15} C_{a} C_{b} d_{a b}^{3} \omega \int_{0}^{\infty} \mathrm{d} r r^{2} \frac{\phi_{a b}}{\partial r} \int_{0}^{r} G_{2}^{a b} \frac{\partial g_{a b}^{0}}{\partial r_{1}} r_{1}^{2} \mathrm{~d} r_{1} \\
\eta_{v}(\omega)= & \left(\rho \frac{N_{0}}{M}\right)^{2} \sum_{a} \sum_{b} \frac{2 \pi}{3} C_{a} C_{b} d_{a b}^{3} \int_{0}^{\infty} \mathrm{d} r r^{3} \frac{\partial \phi_{a b}}{\partial r} \int_{0}^{r} G_{1}^{a b} \stackrel{\leftrightarrow}{\varphi}_{a b} r_{1} \mathrm{~d} r_{1} \\
\eta_{s}(\omega)= & \sum_{a} \frac{\stackrel{k}{P}_{a}(0) \tau_{a}}{\left(1+\left(\omega \tau_{a}\right)^{2}\right)} \\
& +\left(\rho \frac{N_{0}}{M}\right)^{2} \sum_{a} \sum_{b} \frac{2 \pi}{15} C_{a} C_{b} d_{a b}^{3} \int_{0}^{\infty} \mathrm{d} r r^{2} \frac{\partial \Phi_{a b}}{\partial r} \int_{0}^{r} G_{1}^{a b} \frac{\partial \dot{g}_{a b}^{0}}{\partial r_{1}} r_{1} \mathrm{~d} r_{1}
\end{aligned}
$$

where

$$
\begin{aligned}
& \stackrel{k}{P}(0)=n_{a} k T_{0}, \\
& C_{1,2}^{a b}\left(r, r_{1}, \omega\right)= \pm \frac{\tau_{a b}}{2}\left(\frac{2}{\omega \tau_{a b}}\right)\left[\left(\sin \varphi_{1} \mp \cos \varphi_{1}\right) \mathrm{e}^{-\varphi_{1}}-\left(\sin \varphi_{2} \mp \cos \varphi_{2}\right) \mathrm{e}^{-\varphi_{2}}\right], \\
& \tau_{a}=\omega_{a}^{-1}=\frac{m_{a}}{2 \beta_{a}}, \quad \tau_{a b}=\omega_{a b}^{-1}=\frac{d_{a b}^{2}}{k T} \cdot \frac{\beta_{a} \beta_{b}}{\beta_{a}+\beta_{b}}, \\
& \stackrel{*}{\varphi}=\varphi_{a b} / n_{a} n_{b}, \quad \varphi_{1,2}=\left(9 \omega \tau_{a b} / 2\right)^{1 / 2}\left(r \mp r_{1}\right),
\end{aligned}
$$

$K_{s}$ is an adiabatic module of elasticity; $\rho$ is the density of the solution, $N_{0}$ is the Avogadro constant, $M$ is molar mass of the solution, $C_{a}$ and $C_{b}$ are the concentrations of particles of sorts $a$ and $b$, respectively.

Expressions (7)-(10) describe a dynamic behaviour of viscoelastic properties of electrolyte solutions. The frequency dependence of modules of elasticity and factors of viscosity is caused, mainly, by the process of structural relaxation. This dependence is determined by the behaviour of functions $G_{1}^{a b}\left(r, r_{1}, w\right)$ and $G_{2}^{a b}\left(r, r_{1}, w\right)$. The translational and structural relaxations in solutions of electrolytes according to (7)-(10) play unequal role. The relaxation of shear viscosity and shear module of elasticity is both translational and structural, while the relaxation of bulk viscosity and bulk module of elasticity is structural only.

The realization of numeric calculations of the obtained expression (7)-(10) demands a concrete choice of the potential of interparticle interaction and the radial equilibrium distribution function which is considered as known because of (8). 
Let us consider some limiting cases of the obtained results. At low frequencies, when $w \tau \ll 1$, the bulk module of elasticity tends to its adiabatic value $K_{s}$, and shear module of elasticity tends to zero under the law $\omega^{3 / 2}$, and the bulk and shear viscosity coefficients tend to their static values as linear functions of $\omega^{1 / 2}$, which completely agree with the results of works [9,10], obtained using the method of molecular dynamics for liquids.

In a high-frequency regime, when $\omega \tau \gg 1$, the modules of elasticity do not depend on frequency and coincide in the form with high-frequency modules of elasticity obtained by Zwanzig for liquids [11], and the factors of viscosity tend to zero proportionally to $\omega^{-1}$.

Thus, conducted asymptotic evaluations of the obtained results for the solutions of electrolytes completely correspond to general conclusions of the statistical theory of viscoelastic properties of liquids. At slow processes, the expressions (7)-(10) describe viscous properties, and in the case of very fast processes, the same expressions describe the elastic properties of solutions. In a high-frequency region, the liquid behaves as amorphical solid state and besides the bulk module of elasticity, the shear module of elasticity occurs which ensures the possibility of distribution of both longitudinal and transversal acoustic modes in the solutions of electrolytes.

\section{References}

1. Bearman R.J., Kirkwood J.G. // Chem. Phys., 1958, vol. 28, No. 1, p. 136.

2. Naghizadeh J. // Chem. Phys., 1963, vol. 39, No. 12, p. 3406.

3. Haus J.W. // Chem. Phys., 1974, vol. 60, No. 7, p. 2438.

4. Mo K.S., Gubbins K.E. // Mol. Phys., 1976, vol. 31, No. 3, p. 825.

5. Erdey-Grous T. Transport Phenomena in Aqueous Solutions. Moskow, Mir, 1976.

6. Fisher I.Z., Zajtseva A.M. // Dokl. Acad. Nauk SSSR, 1964, vol. 154, p. 1175.

7. Odinaev S., Adchamov A.A. Molecular Theory of Structural Relaxation and Transport Phenomena in Liquids. Dushanbe, Donish, 1998.

8. Yukhnovskii I.R., Holovko M.F. Statistical Theory of Classical Equilibrium Systems. Kyiv, Naukova Dumka, 1980.

9. Lagarkov A.N., Sergeev V.M. // Usp. Fiz. Nauk, 1978, vol. 125, No. 3, p. 409.

10. Evans D.J., Henly C.J., Hess Z. Non-Newtonian phenomena in simple liquids. - In collection "Fizika za rubezhom", Moskow, Mir, 1986, p. 7-28.

11. Zwanzig R., Montain R.D. // Chem. Phys., 1965, vol. 43, No. 12, p. 4464. 


\section{До статистичної теорії в'язкопружних властивостей розчинів електролітів}

\section{С.Одінаєв, А.Додарбеков}

Фізико-технічний інститут Академії наук Республіки Таджикистан, Республіка Таджикистан, 734063 Душанбе, вул. Айні 229/1

Отримано 30 серпня 2000 р.

Отримано аналітичні вирази для динамічних коефіцієнтів зсувної та об'ємної в'язкості та відповідних модулів пружності на основі розв'язку узагальненого кінетичного рівняння для одно- та двочастинкових кореляційних функцій. Частотна дисперсія цих коефіцієнтів і модулів пружності зумовлена в основному трансляційними та структурними релаксаційними процесами. Встановлена асимптотична низько- та високочастотна поведінка отриманих виразів.

Ключові слова: кінетичне рівняння, трансляційна та структурна релаксація, зсувна та об'ємна в'язкість, модулі пружності, розчини електролітів

PACS: $61.20 . \mathrm{Qg}, 66.10 . \mathrm{Ed}$ 